\title{
Biomarker of Dietary Intake
}

National Cancer Institute

\section{Source}

National Cancer Institute. Biomarker of Dietary Intake. NCI Thesaurus. Code C18599.

Dietary induced changes in the serum metabolome which serve as biomarkers for physiological status (e.g., relative risk of developing age-related diseases such as cancer). 